MOUSE MODELS

\title{
Lab mice go native
}

$c 4$

\section{The mouse \\ model}

described by

Rosshart et al. highlights the

importance of considering

the entire

meta-organism

(including

natural

microbiota)

when

assessing drug

responses
Laboratory mice have been instrumental in drug discovery, but there are important limitations to their translational research value. Although these issues are mainly due to the genetic differences between mice and humans, the environmental conditions of lab mice - especially regarding pathogen exposure and microbiota - also have an impact. Now, reporting in Science, Rosshart et al. have generated mice with an immune phenotype and microbiome that resemble those of mice in natural environments, which they show could provide more realistic disease models.

The authors transferred embryos from the most commonly used lab mouse strain (C57BL/6) into pseudopregnant female wild mice that had been paired with vasectomized wild males. The offspring, named 'wildlings', had bacterial, fungal and viral microbiomes that resembled those of wild mice, and that were different to those of lab mice. Compared with lab mice, the gut microbiome in wildlings was stable over multiple generations and resistant to environmental disturbances such as treatment with antibiotics and cohousing experiments. In these experiments - in which mice eat faeces from their cage mates, leading to microbiota exchange - natural gut microbiota remained persistent, whereas the gut microbiome of lab mice was completely replaced by natural microbiota.

The authors also compared the immune landscape in the three mouse groups (lab mice, wild mice and wildlings) by mass cytometry and RNA sequencing. They assessed immunologically important epithelial barriers (gut, skin and vagina), a central nonlymphoid organ (liver) and a central lymphoid organ (spleen) along with blood lymphocytes. "We found that the microbiome affected the immune landscape of barrier sites and the liver. The immune phenotype of wildlings in spleen and blood, which are organs frequently used in human and animal immunological research, was closer to that of wild mice than to lab mice," explains Rosshart.

Finally, the authors tested the translational research value of the wildling mouse model by replicating mouse-based studies that had failed when transitioned to clinical trials in humans. First, they chose the CD28-superagonist (CD28SA) trial. In preclinical studies, treatment with a CD28SA monoclonal antibody (mAb) resulted in activation and expansion of anti-inflammatory regulatory $\mathrm{T}\left(\mathrm{T}_{\mathrm{reg}}\right)$ cells and was effective in several models of inflammatory and autoimmune disease. However, in phase I trials, treatment resulted in an unexpected and severe activation of inflammatory $\mathrm{T}$ cells followed by a deadly cytokine storm. When Rosshart et al. treated wildlings and lab mice with a CD28SA $\mathrm{mAb}$, the number of splenic $\mathrm{T}_{\text {reg }}$ cells increased in lab mice. By contrast, wildlings did not show an increase in $\mathrm{T}_{\text {reg }}$ cell number, but they had higher levels of serum interferon- $\gamma$ (IFN $\gamma$ ), interleukin-1 $\beta$ (IL-1 $\beta$ ), IL-2, IL-4, IL- 6 and tumour necrosis factor (TNF), which replicated the inflammatory response from patients in the trial.

Next, the authors chose a trial that assessed the efficacy of anti-TNF treatment during septic shock. In preclinical studies, a TNFneutralizing antibody protected mice from death from endotoxaemia after an injection of Escherichia coliderived lipopolysaccharide. However, TNF blockade did not reduce the mortality of patients with septic shock; furthermore, it seemed to be associated with increased mortality, which led to the early termination of the study. When Rosshart et al. treated the different groups of mice with an anti-TNF mAb after induction of lethal endotoxaemia, lab mice had a $54 \%$ higher survival rate compared with control mice. However, treatment did not increase the survival rate of wildlings, which was lower than in the control group, replicating the effects observed in patients in the clinical trial.

The mouse model described by Rosshart et al. highlights the importance of considering the entire meta-organism (including natural microbiota) when assessing drug responses. "The use of these mice could have prevented two major failed clinical trials, thereby protecting humans from harm, and saved a lot of money," says Rosshart.

The team is now working on optimizing and assessing wildling mouse models to identify protective mechanisms and potential targets for drug development in areas such as infectious diseases, inflammatory diseases and cancer immunotherapy.

M. Teresa Villanueva

ORIGINAL ARTICLE Rosshart, S. P. et al. Laboratory mice born to wild mice have natural microbiota and model human immune responses. Science 365, eaaw4361 (2019) 